\title{
Laser drilling improving circuit board manufacturing
}

\author{
YU Gang ${ }^{1}$ ZHANG Jincheng GAO Chunlin WANG Hongcai LI Ping AN Yongqiang ZHANG Guiqiu \\ Institute of Mechanics, Chinese Academy of Sciences, Beijing 100080 China
}

\begin{abstract}
We reported here a novel technique for laser high speed drillings on Printed Circuit Boards (PCBs). A CNC solid laser based system is developed to drill through and blind vias as an alternative to mechanical drilling. The system employs an AcoustoOptic Q-switched Nd: YAG laser, a computer control system and an X-Y moving table which can handle up to $400 \times 400 \mathrm{~mm}$ PCB. With a special designed cavity the laser system works in a pulsed operation in order to generate pulses with width down to $0.5 \mu \mathrm{s}$ and maximum peak power over $10 \mathrm{~kW}$ at 10k repetition rate. Delivered by an improved optical beam transforming system, the focused laser beam can drill holes including blind vias on PCBs with diameter in the range of $0.1-0.4 \mathrm{~mm}$ and at up to $300-500$ vias per second (depending on the construction of PCBs). By means of a CNC X-Y moving system, laser pulses with pulse-to-pulse superior repeatability can be fired at desired location on a PCBs with high accuracy. This alternative technology for drilling through or blind vias on PCBs or PWBs (printed wiring boards) will obviously enhance the capability to printed boards manufacturing.
\end{abstract}

Keywords: laser drilling, printed circuit boards

\section{BACKGROUND}

Electrical connections between layers of $\mathrm{PCB}$ have conventionally been achieved by drilling holes and then plating in the board. Mechanical, numerically controlled drills are the standard tool for this work in current industrial practice. However, mechanical drilling is a very capital and labor-intensive process and often a bottleneck in board production. For instance, an advanced technology board today might contain 30-40 thousand holes with diameters down to $0.2 \mathrm{~mm}$, and a single drilling machine (mechanical) may be capable of producing only 5 holes/second ${ }^{[1]}$ In particular, as the diameter of a hole to be drilled decreases, an expensive ultrahard drill having a high physical rigidity must be used. General speaking, small drills have a fairly limited useful lifetime, because even a small amount of drill damage can lead to instability and drill breakage. It is evident that the mechanical drilling operation is often the limiting factor in board throughput. ${ }^{[1]}$

In recent years, laser has shown its great potentialities being as a useful tool in drilling small holes with high speed on printed

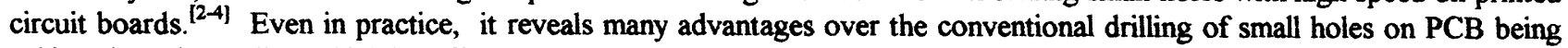
achieved mechanically, which is suffering from low speed and labor-intensed. Several attempts approaching the fast and fine laser drillings on PCBs or PWBs have been made. Kunio Arai described an apparatus consisting of a drill head to perforate a pilot hole on upper copper foil and a laser beam to get rid of the intermediate resin layer of lower copper foil for multi-layer board hole drillings. ${ }^{\left[{ }^{2]}\right.}$ Hideho Inagawa demonstrated a method to form no-conical shape holes on PCBs by using two laser beams (pulse and wavelength differed) and adjusting the focus lens position. ${ }^{[4]}$ More recently a TEA $\mathrm{CO}_{2}$ laser is used for high speed blind vias drilling on PCBs or PWBs. ${ }^{[5]}$ Here we report a novel technique of laser drilling for high speed and accuracy hole makings on PCBs or PWBs. It employs a Q-switched solid state (YAG) laser with which a required laser pulse trains can be achieved for drilling through and blind vias, and an industrial computer system to control both laser pulse parameters and $\mathrm{X}-\mathrm{Y}$ table movements. Benefit from a shorter wavelength (compared with $10.6 \mu \mathrm{m}$ of a $\mathrm{CO}_{2}$ laser) of YAG beam and an Acousto-Optic Q-switched technique, our solid state CNC laser drilling installation provides more attractive features for industry, such as higher vias forming speed, easier to control and maintenance, and lower cost for the equipment. ${ }^{[6]}$

\footnotetext{
${ }^{1}$ Primary author contact information: Tel: 861062521859 , Fax: 861062561284 , email: gangyu@public.east.cn.net
} 


\section{PRINCIPLE AND APPARATUS}

Like laser cutting and welding, laser perforating on one material has a long history of industrial usage. However, drilling on PCB which is constructed by few material layers is a different job. Figure 1 shows a typical PCB structure in cross section view, in Figure 1 (a) is a single type and in Figure 1 (b) is a multi type. On both sides of PCB are copper foils, the thickness of metal layer varies from $35 \mu \mathrm{m}$ to $70 \mu \mathrm{m}$. And in between is the isolated resin layer with glass fiber bundles for enhancing the strengths. Therefore drilling through or blind vias on PCB is a complex processing: the amounts of removal of the copper layers, the resin layers and the glass fiber layers are not equal because of various factors such as energy absorption capacity, absorption coefficient, energy density and irradiation time, etc.

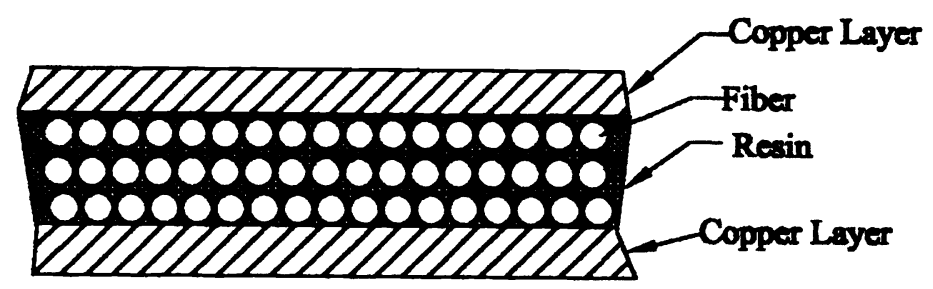

(a) single type PCB

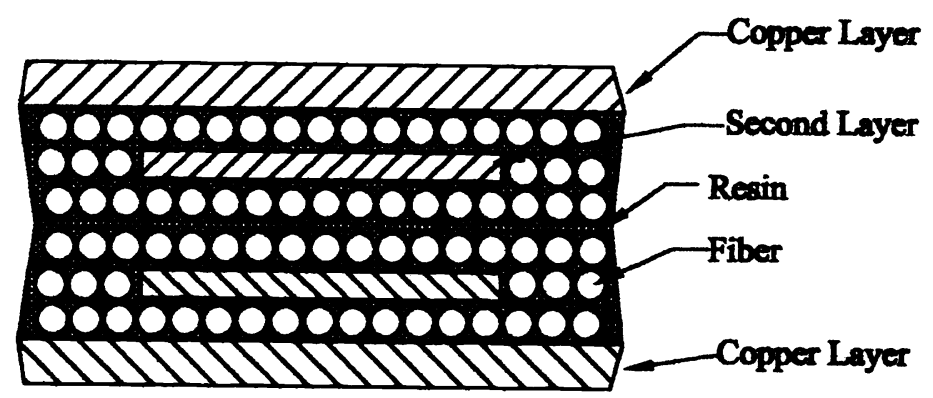

(b) multi type PCB

Fig. 1 Cross-section view of construction of PCBs.

The process may be divided approximately into three steps, as illustrated in Figure 2 Firstly, a train of laser pulses are focused and irradiated onto the upper surface of a PCB, the affected area of the front copper foil is removed by vaporizing due to high energy density of laser pulses, and then a pilot hole is formed, as in Figure 2 (a). After penetrating the upper metal layer, the second group of laser pulses continue to irradiate and making a hole in the mixture portion of resin with glass fibers. This is the key processing of laser drilling on PCBs, as shown in Figure 2 (b) and (c). Finally, repeated as the first step a hole can be formed on the lower copper foil layer of the PCB by the third group of laser pulses depending upon whether a through hole is required, as in Figure 2 (d). The quality and speed of laser perforating are determined by the pulse parameter like energy, width, frequency and group, etc., the optical transmitting and focusing facility, and the CNC mechanical moving ability.

Figure 3 presents a schematic diagram of a computer controlled laser drilling system for PCBs or PWBs. A Nd:YAG is used to emit $1.06 \mu \mathrm{m}$ laser beam, and the c.w. output is converted into pulsed mode by employing an A-O Q-switch within the laser cavity. With a special designed cavity the laser system generates good beam quality pulses with width down to $0.5 \mu$ s and maximum peak power over $10 \mathrm{~kW}$ at $10 \mathrm{kHz}$ repetition rate. The laser rod is excited by two discharge lamps at same time using a $6 \mathrm{~kW}$ power supply. A two stage recirculating water cooling system (the inner circulation employs a deionization water) 


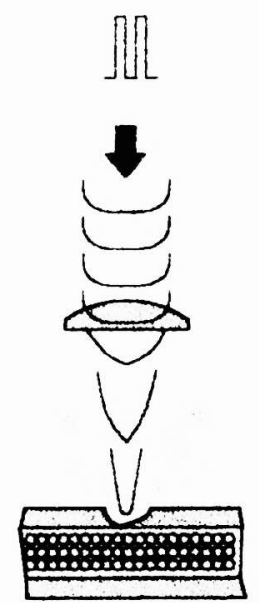

(a)

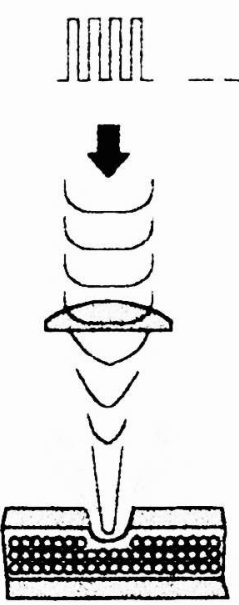

(b)

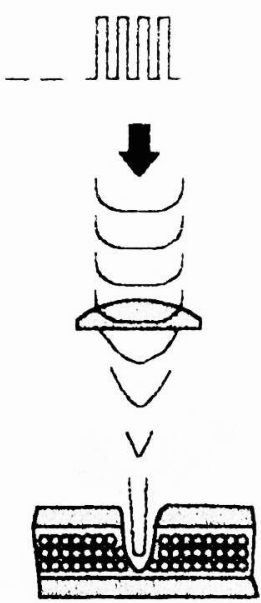

(c)

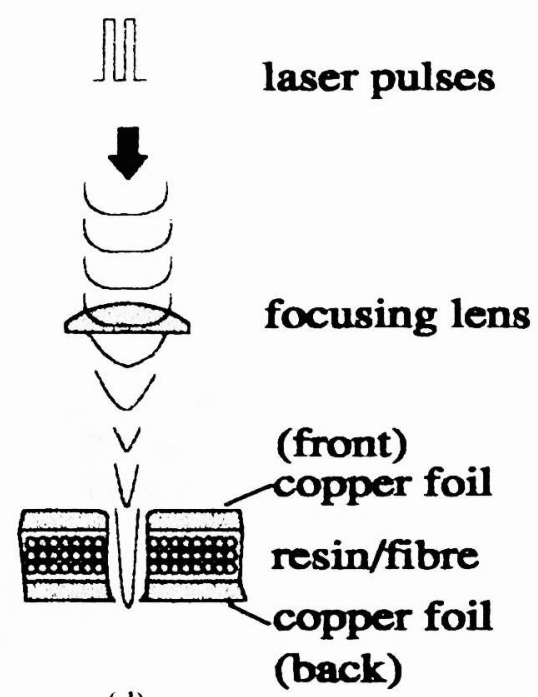

(d)

Fig.2 Process of laser drilling on PCB
(a) drilling pilot hole on front copper layer
(b) (c ) drilling hole on resin and fiber
(d) drilling hole on rear copper layer

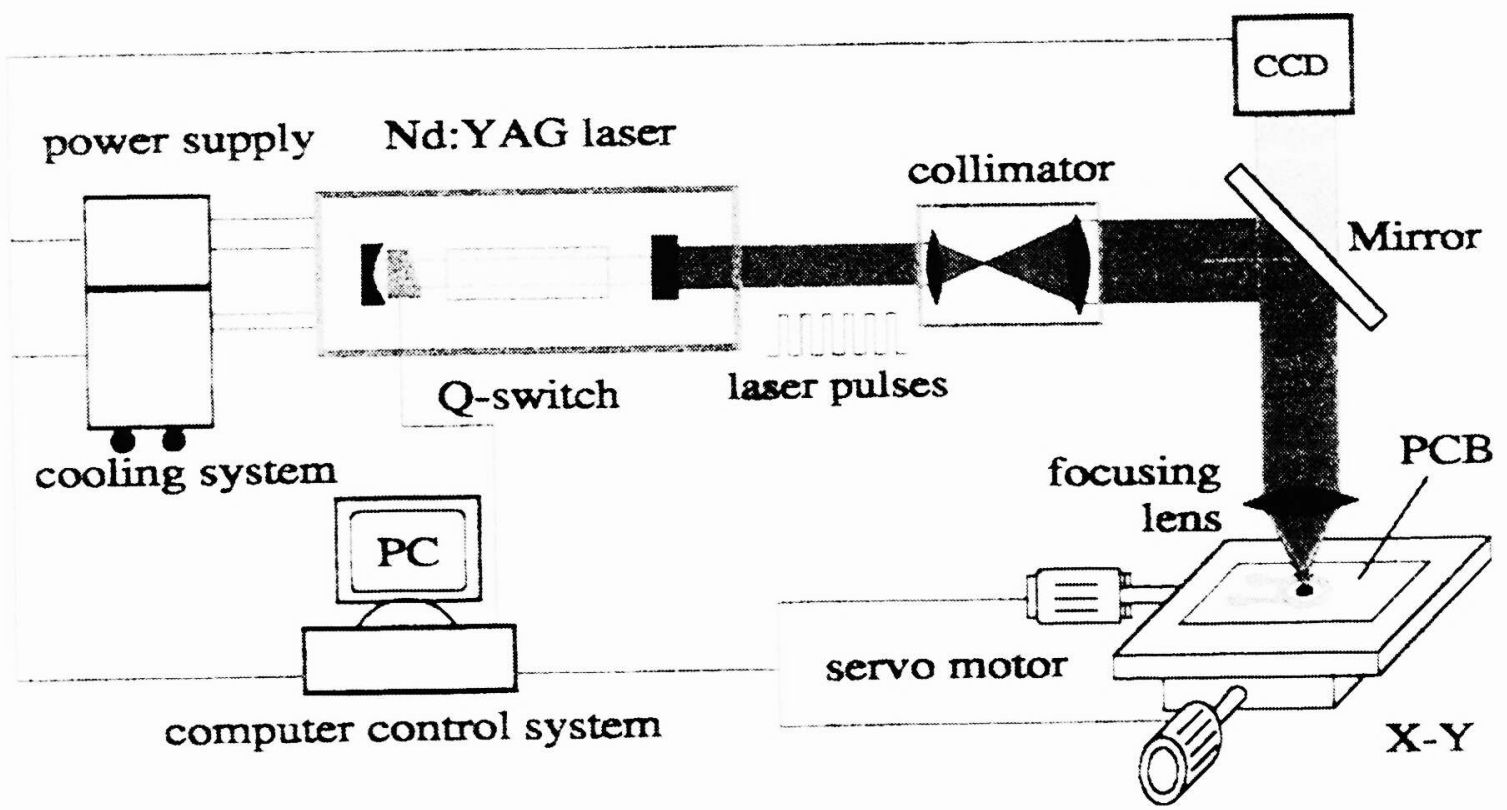

Fig. 3 Diagram of $\mathrm{CNC}$ laser drilling apparatus 
removes the waste heat. Laser pulses are delivered by optical transforming unit onto the surface of workpieces. The unit has a $\times 5$ beam expander to reduce the beam divergence and then the focal spot size. Combination with the transverse laser mode control and optical beam focusing. the drilling hole diameter can be pre-determined. An aiding gas ejected through the nozzle with a certain shape can protect the optics from contamination and also improve the working speed and quality of holes. A CCD camera is used to monitor the accomplishment of drilling process, and the image can be displayed on a PC with Microsoft Windows'95 platform. Instead of turning the laser beam focusing unit to fix the hole drilling position, the X-Y table having computer controlled servo-motors as moving source provides a fast and accurate hole locations. All controls including laser pulse parameters, drilling board information, and hole locations are programmable and realized with only one industrial PC

\section{RESULTS AND ANALYSIS}

The PCBs used in our experiments are $0.2 \mathrm{~mm}$ and $0.4 \mathrm{~mm}$ in total thickness, respectively, which are commonly used in printed board industry. The copper foil layers of both boards are $35 \mu \mathrm{m}$. When the laser pulse parameters are set after a trial drilling for different layers, pulses in group are fired onto the above mentioned boards.

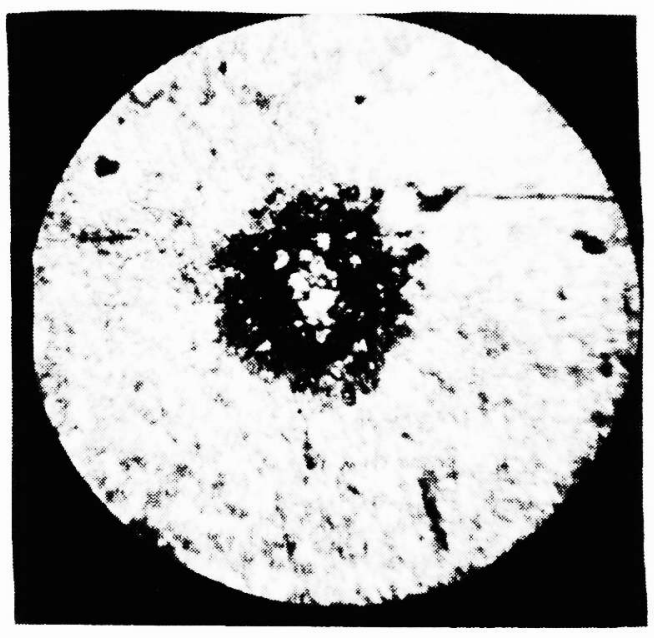

(a) result of 4 pulses

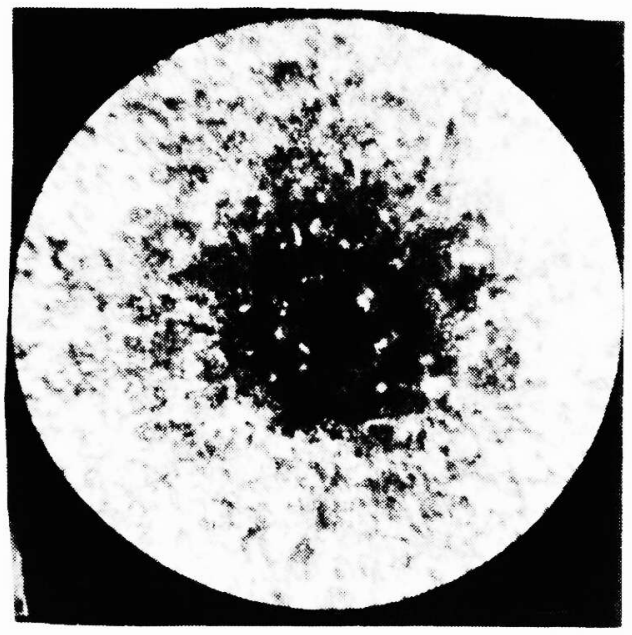

(c) result of 14 pulses

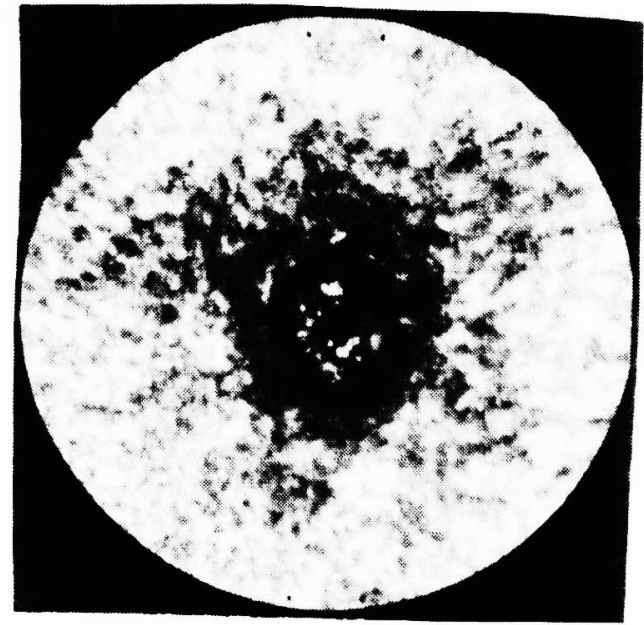

(b) result of 10 pulses

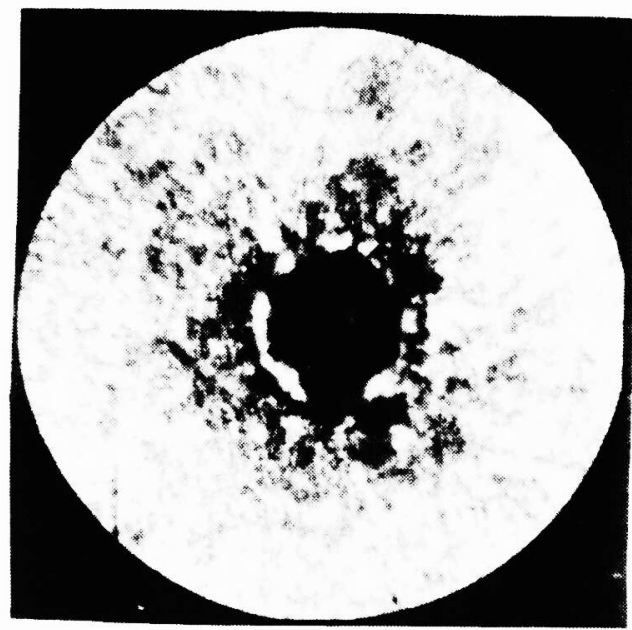

(d) result of 18 pulses

Fig 4 Laser drilling processing using different pulse trains 
Figure 4 clearly shows the result of the processing when different group of laser pulses are shot on a PCB. The experiment conditions are: energy per pulse $8 \mathrm{~mJ}$, pulse width: $0.5 \mu \mathrm{s}$, pulse repetition rate: $6 \mathrm{k}$, beam expander: 5 times, focal length: $80 \mathrm{~mm}$, and number of pulses 4 in (a), 10 in (b), 14 in (c) and 18 in (d). If a pulse train contains pulses less than ten, no pilot hole can be formed, only heat affected zone takes shape, see (a) and (b) for example. When pulses in the train raised over 16 , the pilot hole distinctly appears, as shown in (d). The diameter of the pilot hole is about $150 \mu \mathrm{m}$ in (d). The total energy required to remove the upper copper layer and to drill a desirable pilot hole is the sum of 16-18 pulse. It may take less than one milli-second if the frequency of pulsed operation increased. Immediately after the penetrating of the upper copper layer, next pulse train poccessing different parameters are set to irradiate onto the resin layer and glass fibers further through the pilot hole, when this portion of PCB is removed a key via is formed. If a through hole of a PCB is required, the third group of pulses with the same information of first train are needed to fire on the bottom copper layer, and then the hole drilling is finished. If a blind hole is required for a multi-layer PCB (more than three copper layers), only first train (for copper layer) and second train (for resin and fiber) of pulses are used repeatedly until the certain layer drilling completed, leaving the next copper foil undamaged for electric connections

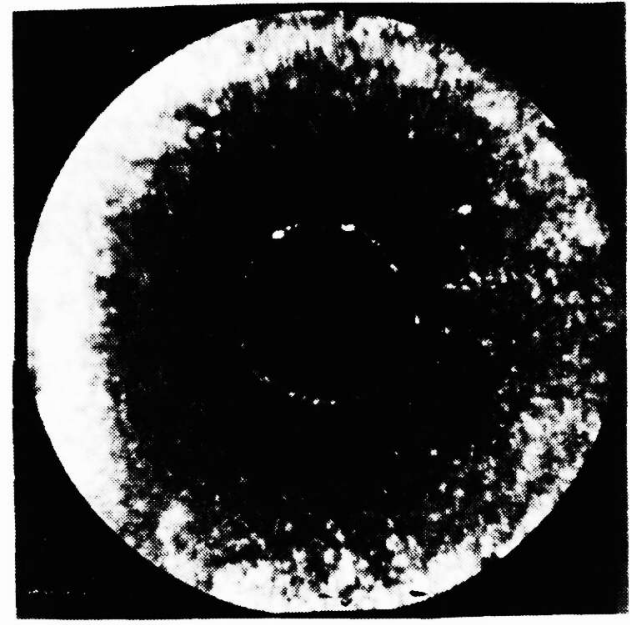

(a)

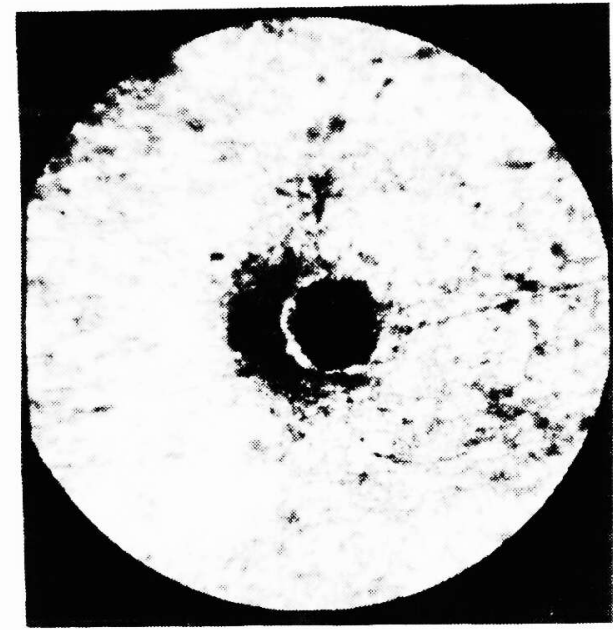

(c)

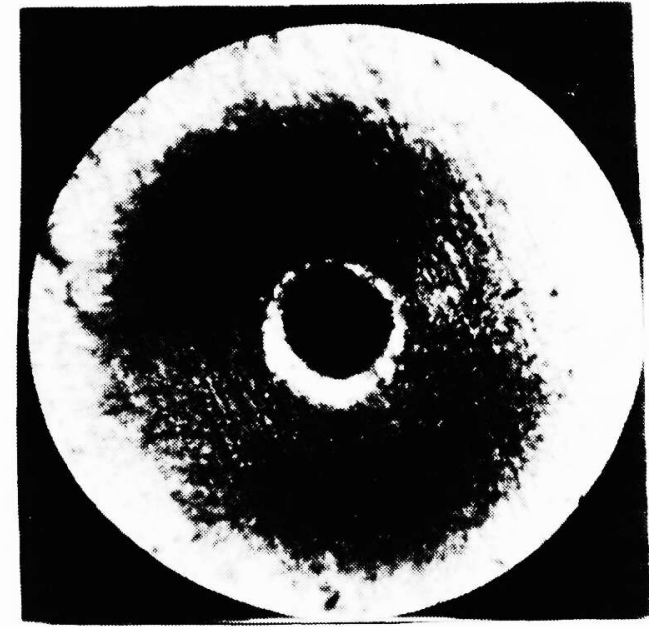

(b)

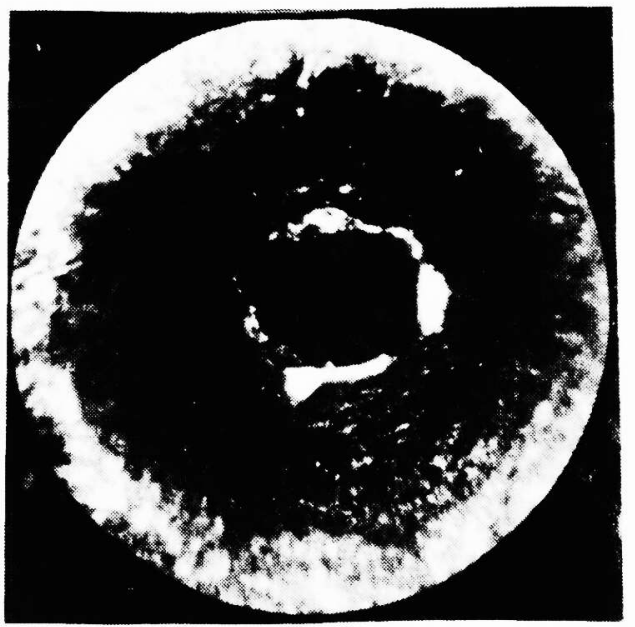

(d)

Fig 5 Typical photographic views of laser drilled holes on PCBs

(a) the hole with a diameter of $170 \mu \mathrm{m}$ on the front layer

(b) the hole with diameter of $120 \mu \mathrm{m}$ on the back layer

(c) the hole with diameter of $90 \mu \mathrm{m}$ on the back layer

(d) a "bad" hole with carbonization effect 
Figure 5 shows a typical photographic views of laser drilled holes on PCBs. Figure 5 (a) is the front hole with a diameter of 170 $\mu \mathrm{m}$. Figure 5 (b) and (c) are the rear holes with diameters of 120 and $90 \mu \mathrm{m}$, respectively. The size of a back hole can be determined by fixing the location of focus along optical axis according to propagation characteristics of a focused Gaussian beam. This may provide a feature to laser-drill a shaped hole, which can not be easily carried out by using other techniques. If operation parameters are not set correctly, in particular, if the laser pulse parameters do not match the PCB or vice versa, a "bad" hole may be formed, as shown in Figure 5 (d). This is mainly due to the carbonization effect by low energy over heating and off the focus. Not only the hole size is out of control but the wall of the hole (even the hole edge on copper foil) is not clean as well. In order to get a "good quality" hole a special designed laser cavity must have the following peculiarities: a controlled transverse mode, a reduced pulse duration (width), a high peak power, a high repeatability of pulse-to-pulse and a long cooling time between pulses. ${ }^{[6]}$ It is also important that these pulses are delivered onto the working surface of a PCB by a carefully constructed laser beam transforming and focusing unit. finally, a high flow rate aiding gas ejecting from a shaped nozzle is used to blow away all the waste materials during the drilling process and to reduce the heat affected zone (HAZ) formed between the irradiated pulses. The flow gas supplies a protection of optics from damage too.

Since a computer control technology is introduced, the pulse train parameter, the PCB or PWB board information, and the hole penetration procedure can be stored and programmed, this leads to an automatic fabrication of PCB hole drillings. By means of CAD (computer aided design) and CAM (computer aided manufacture) concepts, the laser drilling processing on PCBs including through holes and blind holes may be optimized, resulting the highest speed, the top quality and repeatability of PCB hole makings, which offers a room for the improvement of PCB manufacturing.

\section{CONCLUSION}

An advanced laser drilling technique on PCBs has been studied above. It has several advantages for industrial application, named mainly here: the drilling is a non-contact, minimum deformation, high speed and high accuracy processing. A laser drilling apparatus has also been demonstrated for an industrial practice. The improved laser beam characters, pulse parameters and together with the computer control technique will provide an excellent appearance of hole drillings on PCBs or PWBs. It is certain that laser drilling can enhance the ability of printed circuit board production.

\section{ACKNOWLEDGMENTS}

The authors would like to thank C.A.S. ( Chinese Academy of Sciences) and the Ministry of Labor and Personnel Affairs for their financial support, especially the funding for Returned Scholars and Students Studied from Western Countries. Without their help the experiments can not be carried out.

\section{REFERENCES}

1. James S. Arzigian, DE94011004/HDM, US G. Report, May, 1994.

2. Kunio Arai and Yasuhiko Kanaga, US Patent 5010232, Apr., 1991.

3. Hideho Inagawa and Shigenobu Nojo, US Patent 5063280, Nov., 1991.

4. Hideho Inagawa and Shigenobu Nojo, US Patent 5073687, Dec., 1991.

5. Report, Industrial Laser Review, Nov., 1996.

6. G. Yu, J.C. Zhang, H.C. Wang, C.L. Gao, Y.J. Hu, P. Li, and M. Shi, submitted to Chinese Patent, applying number: 98203943.3. Apr. 1998 